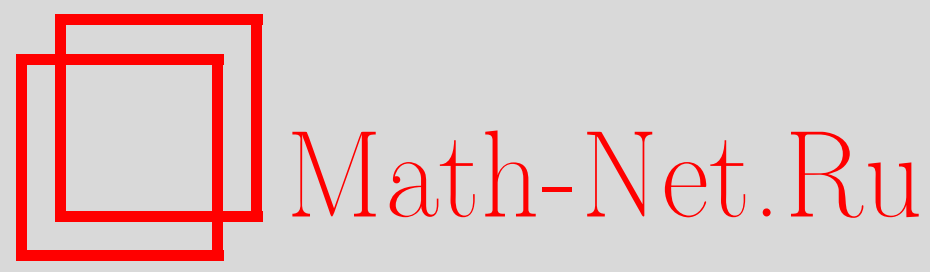

О. П. Филатов, Асимптотический метод в задачах вычисления пределов максимальных средних, Матем. заметки, 1999, том 66, выпуск 3, 431-438

DOI: https://doi.org/10.4213/mzm1184

Использование Общероссийского математического портала Math-Net.Ru подразумевает, что вы прочитали и согласны с пользовательским соглашением http://www . mathnet.ru/rus/agreement

Параметры загрузки:

IP: 34.229 .108 .108

26 апреля 2023 г., 16:10:54 


\section{АСИМПТОТИЧЕСКИЙ МЕТОД В ЗАДАЧАХ \\ ВЫЧИСЛЕНИЯ ПРЕДЕЛОВ МАКСИМАЛЬНЫХ СРЕДНИХ}

\section{О.П. Филатов}

Для непрерывной почти периодической функции $f: \mathbb{R}^{m_{\tau}} \times \mathbb{R}^{m_{\gamma}} \rightarrow \mathbb{R}$ установлено, что функция

$$
M_{f}(\mu)=\lim _{\Delta \rightarrow \infty} \sup _{\tau, \gamma} \frac{1}{\Delta} \int_{0}^{\Delta} f(\tau(\mu t), \gamma(t)) d t,
$$

где точная верхняя грань берется по всем решениям системы дифференциальных включений $\dot{\tau} \in \mu G_{\tau}, \tau(0)=\tau_{0}, \dot{\gamma} \in G_{\gamma}, \gamma(0)=\gamma_{0}$, при $\mu \rightarrow+0$ имеет предел

$$
\Psi_{f}=\lim _{\Delta \rightarrow \infty} \sup _{\tau} \frac{1}{\Delta} \int_{0}^{\Delta} \Phi(\tau(\mu t)) d t, \quad \text { где } \Phi\left(\tau_{0}\right)=\lim _{\Delta \rightarrow \infty} \sup _{\gamma} \frac{1}{\Delta} \int_{0}^{\Delta} f\left(\tau_{0}, \gamma(t)\right) d t .
$$

Таким образом, $\Psi_{f}=\lim _{\mu \rightarrow+0} M_{f}(\mu)$ и предел максимального среднего приближенно можно находить решая задачи меньшей размерности, если параметр $\mu$ мал. При этом, если компакты $G_{\tau} \subset \mathbb{R}^{m_{\tau}}, G_{\gamma} \subset \mathbb{R}^{m_{\gamma}}$ являютя невырожденными, то $\Psi_{f}$ не зависит от начальных данных.

Библиография: 3 названия.

1. Постановка задачи. Линейное пространство непрерывных почти периодических функций $f: \mathbb{R}^{m_{\tau}} \times \mathbb{R}^{m_{\gamma}} \rightarrow \mathbb{R}$, где $\mathbb{R}^{m_{\tau}}$ и $\mathbb{R}^{m_{\gamma}}$ евклидовы пространства, будем обозначать $\Pi\left(\mathbb{R}^{m_{\tau}} \times \mathbb{R}^{m_{\gamma}}\right)$. Для одной из таких функций рассмотрим предел

$$
M_{f}(\mu)=\lim _{\Delta \rightarrow \infty} \sup _{\tau, \gamma} \frac{1}{\Delta} \int_{0}^{\Delta} f(\tau(\mu t), \gamma(t)) d t
$$

где точная верхняя грань берется по всем решениям в смысле Каратеодори системы дифференциальных включений

$$
\begin{array}{ll}
\dot{\tau} \in \mu G_{\tau}, & \tau(0)=\tau_{0}, \\
\dot{\gamma} \in G_{\gamma}, & \gamma(0)=\gamma_{0} .
\end{array}
$$

Здесь $G_{\tau}$ и $G_{\gamma}$ непустые компактные множества соответственно из пространств $\mathbb{R}^{m_{\tau}}$ и $\mathbb{R}^{m_{\gamma}}$. Параметр $\mu>0$ характеризует разнотемповость переменных $\tau$ и $\gamma$.

Работа выполнена при финансовой поддержке Российского фонда фундаментальных исследований, грант № 96-01-00616. 
Множество всех решений задач (2), (3), определенных в промежутке $t \geqslant 0$, будем обозначать соответственно $\Gamma_{\tau}\left(\tau_{0}\right), \Gamma_{\gamma}\left(\gamma_{0}\right)$.

В данной работе ставится задача нахождения предельного значения $\lim _{\mu \rightarrow+0} M_{f}(\mu)$. Установлено, что указанный предел можно вычислять, решая последовательно задачи меньшей размерности соответственно для дифференциальных включений (3) и (2). Поскольку в одномерном случае пределы максимальных средних можно вычислять достаточно эффективно [1], полученные результаты позволяют приближенно находить решения и в многомерной ситуации для разнотемповых переменных. Особенно удобен такой прием при $m_{\tau}=m_{\gamma}=1$.

С точки зрения приложений к теории усреднения дифференциальных включений обычно существенно, чтобы предел максимальньх средних $M_{f}$ не зависел от начальных условий. Тем не менее, мы будем рассматривать поставленную задачу в более общей постановке для таких компактов $G_{\tau}$ и $G_{\gamma}$, которые гарантируют независимость хотя бы предельного значения $\lim _{\mu \rightarrow+0} M_{f}(\mu)$ от начальных векторов $\tau_{0}, \gamma_{0}$.

2. Основные обозначения и определения. Пусть $C\left(\mathbb{R}^{m}\right)$-пространствонепрерьвных функций $f: \mathbb{R}^{m} \rightarrow \mathbb{R}$ с равномерной метрикой, $f_{h}(\gamma)=f(\gamma+h)$. Напомним, что функция $f \in C\left(\mathbb{R}^{m}\right)$ назьвается почти периодической (по Бохнеру), если для произвольной последовательности векторов $h_{n} \in \mathbb{R}^{m}, n=1,2, \ldots$, семейство функций $f_{h_{n}}$ будет предкомпактным.

Далее нам потребуется и эквивалентное определение почти периодичности по Бору: функция $f \in C\left(\mathbb{R}^{m}\right)$ называется почти периодической, если для любого $\varepsilon>0$ существует число $l=l(\varepsilon)>0$ такое, что любой $\left(m\right.$-мерный) куб из $\mathbb{R}^{m}$ с длиной стороны равной $l$, содержит векторный $\varepsilon$-почти период $T_{\varepsilon}$ функции $f$, т.е. при любом $\gamma \in \mathbb{R}^{m}$ выполняется неравенство $\left|f\left(\gamma+T_{\varepsilon}\right)-f(\gamma)\right| \leqslant \varepsilon$.

Для того чтобы предельное значение $\lim _{\mu \rightarrow+0} M_{f}(\mu)$ не зависело, причем равномерно, от начальных данных $\tau_{0} \in \mathbb{R}^{m_{\tau}}, \gamma_{0} \in \mathbb{R}^{m_{\gamma}}$, на компакты $G_{\tau}, G_{\gamma}$ требуется наложить условия невырожденности в смысле следующего определения: компакт $K \subset \mathbb{R}^{m}$ называется $(m-1)$-невырожденным, если его размерность $\operatorname{dim}(K) \geqslant m-1$; при этом, если $\operatorname{dim}(K)=m-1$, то компакт не должен принадлежать подпространству размерности $m-1$. Для краткости будем говорить просто о невырожденности компакта $K$ в условиях данного определения.

Например, в случае $m=2$ компакт $K \subset \mathbb{R}^{2}$ будет невьрожденньм тогда и только тог да, ког да множество $K$ не принадлежит прямой, проходящей через начало координат.

Если компакты $G_{\tau} \subset \mathbb{R}^{m_{\tau}}$ и $G_{\gamma} \subset \mathbb{R}^{m_{\gamma}}$ являются невырожденньми, то прямое произведение $G=G_{\tau} \times G_{\gamma}$ может оказаться и вырожденным. Например, если $m_{\tau}=1$, $m_{\gamma}=2, G_{\tau}=\{1\}, G_{\gamma}=\{1\} \times[1,2]$, то множество $G \subset \mathbb{R}^{3}$ - вырожденное. В то же время невырожденность прямого произведения $G_{\tau} \times G_{\gamma}$ влечет невырожденность множеств $G_{\tau}$ и $G_{\gamma}$

Сразу же отметим, что если предел максимального среднего существует равномерно по начальным условиям, то предел реализуется на некотором решении дифференциального включения. Этот факт (обоснованный в предыдущей публикации) мы будем использовать при доказательстве основной теоремы.

Модуль непрерьвности функции $f(\tau, \gamma)$ по переменным $\tau \in \mathbb{R}^{m_{\tau}}$, вычисляемьй по всем шарам пространства $\mathbb{R}^{m_{\tau}}$ радиуса $\delta>0$, будем обозначать $\omega_{\tau}(\delta, f)$. Постоянная $f_{0}=\sup _{\tau, \gamma}|f(\tau, \gamma)|$. Модуль множества $G_{\tau} \subset \mathbb{R}^{m_{\tau}}$ обозначается $\left\|G_{\tau}\right\|=\sup _{\gamma \in G_{\tau}}\|\gamma\|$, где $\|\cdot\|$ - евклидова норма в $\mathbb{R}^{m_{\tau}}$ (а также и в пространстве $\mathbb{R}^{m_{\gamma}}$ ). 
3. Основные результаты. Если $f \in \Pi\left(\mathbb{R}^{m_{\tau}} \times \mathbb{R}^{m_{\gamma}}\right)$, то функция

$$
\Phi\left(\tau_{0}\right)=\lim _{\Delta \rightarrow \infty} \sup _{\gamma} \frac{1}{\Delta} \int_{0}^{\Delta} f\left(\tau_{0}, \gamma(t)\right) d t
$$

где супремум берется по всем решениям $\gamma \in \Gamma_{\gamma}\left(\gamma_{0}\right)$, будет почти периодической, что следует из определения Бохнера. Следовательно, имеет смысл повторньй предел максимального среднего

$$
\Psi_{f}=\lim _{\Delta \rightarrow \infty} \sup _{\tau} \frac{1}{\Delta} \int_{0}^{\Delta} \Phi(\tau(\mu t)) d t .
$$

Здесь супремум берется по всем решениям $\tau \in \Gamma_{\tau}\left(\tau_{0}\right)$. Заметим, что предел $\Psi_{f}$ не зависит от параметра $\mu$.

TЕоремА. Пусть функиия $f \in \Pi\left(\mathbb{R}^{m_{\tau}} \times \mathbb{R}^{m_{\gamma}}\right)$, а компакты $G_{\tau} \subset \mathbb{R}^{m_{\tau}} u G_{\gamma} \subset \mathbb{R}^{m_{\gamma}}$ являются невырожсдеными. Тогда равномерно по начальным условиям $\tau_{0} \in \mathbb{R}^{m_{\tau}}$,

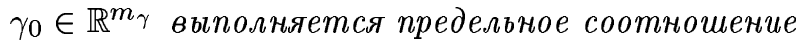

$$
\lim _{\mu \rightarrow+0} M_{f}(\mu)=\Psi_{f}
$$

при этом $\Psi_{f}$ не зависит от начальных данных.

4. ДоКАЗАТЕЛЬСТВо ТЕОРЕМЫ. Оно основьвается на нескольких леммах.

Лемма 1. Если компакт $K \subset \mathbb{R}^{m}$ является невырожденным, а функиия $f \in$ $\Pi\left(\mathbb{R}^{m}\right)$, то для любого $\varepsilon>0$ существует такая постоянная $t_{\max }=t_{\max }(\varepsilon, K)>0$, что для любых точек $a, b \in \mathbb{R}^{m}$ найдется число $t_{a} \in\left[0, t_{\max }\right]$ и интегральная кривая дифференииального включения $\dot{\gamma} \in K$, соединяющая точку $(0, b)$ с точкой $\left(t_{a}, a+T_{\varepsilon}\right) \in \mathbb{R}^{m+1}$ для некоторого в-почти периода $T_{\varepsilon}$ функиии $f$.

ДоКАЗАТЕЛЬСТво ЛЕммы 1. Для Задачи $\dot{\gamma} \in K, \gamma(0)=b$, на основании свойств интегралов от многозначных отображений [2], множество достижимости в момент времени $t$ равно $b+t \cdot \operatorname{co}(K)$, где $\operatorname{co}(K)$ - вьпуклая оболочка компакта. Поэтому объединение множеств достижимости по всем $t \geqslant 0$ представляет из себя конус $C_{b}$ с вершиной в точке $b$,

$$
C_{b}=\left\{\gamma \in \mathbb{R}^{m}: \gamma=b+t \cdot \operatorname{co}(K), \quad t \geqslant 0\right\} .
$$

Поскольку компакт $K$ является невырожденным, в случае, если размерность компакта $\operatorname{dim}(K)=m-1$, множество $K$ содержится в некоторой гиперплоскости $P$, не проходящей через $0 \in \mathbb{R}^{m}$. По теореме о непустоте относительной внутренности вьпуклого множества [3, теорема 1.5 , с. 202$]$ получаем, что множество $\operatorname{co}(K)$ содержит пересечение гиперплоскости $P$ с некоторым шаром пространства $\mathbb{R}^{m}$ радиуса $r_{0}>0$ с центром в некоторой точке гиперплоскости $b_{0}$, при этом угол между вектором $b_{0}$ и нормальным вектором гиперплоскости $P$ отличен от прямого. В таком случае точка $b_{0}$ является внутренней для конуса $C_{b}$.

Если $\operatorname{dim}(K)=m$, то внутренность множества $\operatorname{co}(K)$ непустая, поэтому в качестве $P$ можно взять гиперплоскость, проходящую через внутреннюю точку $b_{0}$ с нормальным вектором равньм $b_{0}$. 
Таким образом, в любом случае существует ( $m$-мерный) куб $K\left(b_{0}\right) \subset C_{b}$ с центром в точке $b_{0}$ и длиной ребра $r>0$ такой, что $b+K\left(b_{0}\right) \subset C_{b}$. Можно считать, что куб $b+K\left(b_{0}\right)$ принадлежит открытому множеству

$$
L(2)=\left\{\gamma \in C_{b}: \gamma=b+t\left(b_{0}+h\right), 0<t<2,\|h\|<r_{0}, h \in P\right\} .
$$

Куб $b+q K\left(b_{0}\right)$ при любом $q>0$ также принадлежит конусу $C_{b}$, поэтому при $q=l / r$ длина его ребра равна $l$. Здесь $l=l(\varepsilon)>0$ - число из определения почти периодичности функции $f$ по Бору. Обозначим этот куб $K_{a}$. Пусть $T_{\varepsilon}$ некоторьй $\varepsilon$-почти период функции $f$, принадлежащий кубу $K_{a}-a$. Тогда для некоторого вектора $a_{1} \in K_{a}$ выполняется равенство $T_{\varepsilon}=a_{1}-a$. Следовательно, $a+T_{\varepsilon} \in K_{a}$.

Так как по построению $K_{a} \subset L(2 q)$, то некоторая интегральная кривая задачи $\dot{\gamma} \in G$, $\gamma(0)=b$, обязательно пройдет через точку $\left(t_{a}, a+T_{\varepsilon}\right)$ в момент времени $t_{a} \leqslant 2 l / r=$ $t_{\max }$. Постоянная $t_{\max }$ для данного $\varepsilon>0$ определяется только компактом $K$ и не зависит от выбора точек $a, b \in \mathbb{R}^{m}$. Лемма 1 доказана.

В следующей лемме

$$
\varphi(\Delta)=\sup _{\tau_{0} \in \mathbb{R}^{m_{\tau}}}\left|\Phi\left(\tau_{0}\right)-M\left(\tau_{0}, \Delta\right)\right|
$$

где

$$
M\left(\tau_{0}, \Delta\right)=\sup _{\gamma_{0} \in \mathbb{R}^{m_{\gamma}}} \sup _{\gamma} \frac{1}{\Delta} \int_{0}^{\Delta} f\left(\tau_{0}, \gamma(t)\right) d t
$$

а точная верхняя грань берется по всем решениям $\gamma \in \Gamma_{\gamma}\left(\gamma_{0}\right)$.

ЛЕмма 2. Пусть компакт $G_{\gamma} \subset \mathbb{R}^{m_{\gamma}}$ является невырожденным, а функиия $f \in \Pi\left(\mathbb{R}^{m_{\tau}} \times \mathbb{R}^{m_{\gamma}}\right)$. Тогда предел $\Phi$, определяемый (4), существует равномерно nо $\tau_{0} \in \mathbb{R}^{m_{\tau}}, \gamma_{0} \in \mathbb{R}^{m_{\gamma}}$, m.e. $\varphi(\Delta) \rightarrow 0$ nри $\Delta \rightarrow \infty$, при этом $\Phi$ не зависит от $\gamma_{0}$.

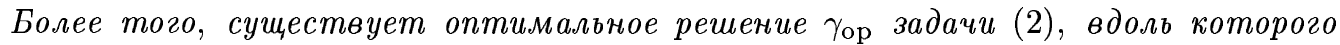
реализуется предел $\Phi$.

ДоКАЗАТЕЛЬСТВо ЛЕмМЫ 2. Обоснование существования предела $\Phi$ на основании леммы 1 проводится аналогично доказательству [1, теорема 2] в одномерном случае. Действительно, в указанной ссылке существенно, что постоянную $t_{\max }=t_{\max }\left(\varepsilon, G_{\gamma}\right)$ (в $\left[1\right.$, теорема 2] она обозначалась $t_{\varepsilon}$ ) из леммы 1 можно выбрать общей для любого параметра $\tau_{0} \in \mathbb{R}^{m_{\tau}}$. Последнее же следует из того, что пространство $\Pi\left(\mathbb{R}^{m_{\tau}} \times \mathbb{R}^{m_{\gamma}}\right)$ является замыканием в равномерной метрике пространства тригонометрических полиномов относительно переменных $\tau \in \mathbb{R}^{m_{\tau}}$ и $\gamma \in \mathbb{R}^{m_{\gamma}}$. Из равномерности существования предела, как уже отмечалось, следует существование оптимального решения. Лемма 2 доказана.

ЛЕмма 3. Пусть $f \in \Pi\left(\mathbb{R}^{m}\right)$. Тогда для любого компакта $K \subset \mathbb{R}^{m}$ предел максимального среднего

$$
M_{f}=\lim _{\Delta \rightarrow \infty} \sup _{\gamma} \frac{1}{\Delta} \int_{0}^{\Delta} f(\gamma(t)) d t
$$

әде супремум берется по всем решениям задачи $\dot{\gamma} \in K, \gamma(0)=\gamma_{0}$, существует равномерно по $\gamma_{0} \in \mathbb{R}^{m}$. Более того, существует оптимальное решение $\gamma_{\text {ор }}$ дифференииального включения, вдоль которого реализуется предел $M_{f}$. 
ДокАЗАТЕЛЬСтво ЛЕммы 3. Если компакт $K$ - невырожденньй, то утверждение следует из леммы 2. Покажем, что и общий случай сводится к основному. Действительно, если компакт $K$ окажется вырожденным, то в простейшем случае $K=\{0\}$ утверждение очевидно. Поэтому считаем $K \neq\{0\}$. Тогда всегда существует собственное подпространство $L \subset \mathbb{R}^{m}$, содержащее множество $K$, размерность которого $\operatorname{dim}(L)=n \geqslant 1$, при этом по отношению к подпространству $L$ само множество $K$ уже является невырожденным. Введем декартовую систему координат, определяемую базисом $e_{1}, e_{2}, \ldots, e_{m}$ пространства $\mathbb{R}^{m}$, где векторы $e_{n+1}, \ldots, e_{m}$ ортогональны к подпространству $L$, а векторы $e_{1}, e_{2}, \ldots, e_{n}$ образуют базис в $L$.

В новых координатах функция $f$ будет по-прежнему почти периодической.

Любое решение дифференциального включения, записанное в этих координатах, обладает свойствами: последние $m-n$ координат решения будут постоянными в силу дифференциального включения $\dot{\gamma} \in K$. Следовательно, их можно рассматривать как параметры в задаче вычисления предела максимального среднего. Поэтому по отношению к координатному пространству $\mathbb{R}^{n}$ опять можно воспользоваться леммой 2 . Лемма 3 доказана.

На основании леммы 3 обозначим $\tau_{*} \in \Gamma_{\tau}\left(\tau_{0}\right)$ - решение дифференциального включения (2) для которого

$$
\lim _{\Delta \rightarrow \infty} \sup _{\tau} \frac{1}{\Delta} \int_{0}^{\Delta} \Phi(\tau(\mu t)) d t=\lim _{\Delta \rightarrow \infty} \frac{1}{\Delta} \int_{0}^{\Delta} \Phi\left(\tau_{*}(\mu t)\right) d t=\Psi_{f}
$$

где супремум берется по всем решениям $\tau \in \Gamma_{\tau}\left(\tau_{0}\right)$. Положим также $\Delta_{0}=\Delta_{0}(\mu)=$ $1 / \sqrt{\mu}, t_{j}=j \Delta_{0}, j=0,1, \ldots$,

$$
S_{n}(\tau)=\sup _{\gamma} \frac{1}{n} \sum_{j=0}^{n-1} \frac{1}{\Delta_{0}} \int_{t_{j}}^{t_{j+1}} f\left(\tau\left(\mu t_{j}\right), \gamma(t)\right) d t
$$

Здесь точная верхняя грань берется по всем решениям $\gamma \in \Gamma_{\gamma}\left(\gamma_{0}\right) ; \tau \in \Gamma_{\tau}\left(\tau_{0}\right)$ - произвольное решение задачи $(2), n=1,2, \ldots$.

Ниже постоянная $t_{\max }=t_{\max }\left(\varepsilon, G_{\gamma}\right)$ определяется леммой 1 для компакта $G_{\gamma}$ и дифференциального включения $\dot{\gamma} \in G_{\gamma}$. Бесконечно малая функция $\varphi(1 / \sqrt{\mu})$ при $\mu \rightarrow+0$ из леммы 2 вводится равенством (6). Наконец, $\delta_{\mu}=\left\|G_{\tau}\right\| \sqrt{\mu}$.

ЛЕмма 4. Пусть компакты $G_{\gamma} \subset \mathbb{R}^{m_{\gamma}} u G_{\tau} \subset \mathbb{R}^{m_{\tau}}$ являются невырожденными, а функиия $f \in \Pi\left(\mathbb{R}^{m_{\tau}} \times \mathbb{R}^{m_{\gamma}}\right)$. Тогда для любого $\varepsilon>0$ существует такое $n_{1}=n_{1}(\varepsilon, \mu)>0$, что если $n \geqslant n_{1}$, то при любых $\tau_{0} \in \mathbb{R}^{m_{\tau}}, \gamma_{0} \in \mathbb{R}^{m_{\gamma}}, \mu>0 u$ любом решении $\tau \in \Gamma_{\tau}\left(\tau_{0}\right)$ выполняется оченка сверху

$$
S_{n}(\tau) \leqslant \Psi_{f}+\omega_{\tau}\left(\delta_{\mu}, f\right)+\varphi\left(\frac{1}{\sqrt{\mu}}\right)+\varepsilon
$$

для решения $\tau_{*} \in \Gamma_{\tau}\left(\tau_{0}\right)$ из (11c)) имеет место оценка снизу

$$
S_{n}\left(\tau_{*}\right) \geqslant \Psi_{f}-2 f_{0} t_{\max } \sqrt{\mu}-\omega_{\tau}\left(\delta_{\mu}, f\right)-\varphi\left(\frac{1}{\sqrt{\mu}}\right)-3 \varepsilon .
$$


ДоКАЗАТЕЛЬСТВО ЛЕмМЫ 4. Для обоснования оценки сверху воспользуемся неравенством

$$
S_{n}(\tau) \leqslant \frac{1}{n} \sum_{j=0}^{n-1} M\left(\tau_{j}, \Delta_{0}\right)
$$

где $\tau_{j}=\tau\left(\mu t_{j}\right)$. Отсюда согласно (6) получим

$$
S_{n}(\tau) \leqslant \frac{1}{n} \sum_{j=0}^{n-1}\left(\Phi\left(\tau_{j}\right)+\varphi\left(\frac{1}{\sqrt{\mu}}\right)\right)=\frac{1}{n \Delta_{0}} \sum_{j=0}^{n-1} \Phi\left(\tau_{j}\right) \Delta_{0}+\varphi\left(\frac{1}{\sqrt{\mu}}\right) .
$$

На отрезке $t_{j} \leqslant t \leqslant t_{j+1}$ вьполняется неравенство $\left|\tau(\mu t)-\tau_{j}\right| \leqslant \delta_{\mu}$, поэтому при любом натуральном $n$ вьполняется оценка

$$
\left|\frac{1}{n \Delta_{0}} \sum_{j=0}^{n-1} \Phi\left(\tau_{j}\right) \Delta_{0}-\frac{1}{n \Delta_{0}} \int_{0}^{n \Delta_{0}} \Phi(\tau(\mu t)) d t\right| \leqslant \omega_{\tau}\left(\delta_{\mu}, f\right) .
$$

При достаточно большом $n \geqslant n_{1}(\varepsilon, \mu)$ и любых $\tau_{0} \in \mathbb{R}^{m_{\tau}}, \gamma_{0} \in \mathbb{R}^{m_{\gamma}}$ справедливы оценки

$$
\frac{1}{n \Delta_{0}} \int_{0}^{n \Delta_{0}} \Phi(\tau(\mu t)) d t \leqslant \sup _{\tau} \frac{1}{n \Delta_{0}} \int_{0}^{n \Delta_{0}} \Phi(\tau(\mu t)) d t \leqslant \Psi_{f}+\varepsilon,
$$

где супремум берется по всем решениям $\tau \in \Gamma_{\tau}\left(\tau_{0}\right)$.

Из (10)-(12) получим (8).

Для получения оценки снизу выражения $S_{n}\left(\tau_{*}\right)$ построим решение $\gamma_{*} \in \Gamma_{\gamma}\left(\gamma_{0}\right)$ дифферециального включения (3) последовательно на отрезках $t_{j} \leqslant t \leqslant t_{j+1}$. С этой целью для любого $j$ введем функцию $\gamma_{j}$ на отрезке $t_{j} \leqslant t \leqslant t_{j+1}$, которая является решением дифференциального включения (3) с некоторым начальным условием $\gamma_{j}\left(t_{j}\right)=\gamma_{j, 0}$, при этом, согласно (6),

$$
\left|\Phi\left(\tau_{j}\right)-\frac{1}{\Delta_{0}} \int_{t_{j}}^{t_{j+1}} f\left(\tau_{j}, \gamma_{j}(t)\right) d t\right| \leqslant \varphi\left(\frac{1}{\sqrt{\mu}}\right)+\varepsilon,
$$

где $\tau_{j}=\tau_{*}\left(\mu t_{j}\right)$. Пусть теперь постоянная $t_{\max }=t_{\max }\left(\varepsilon, G_{\gamma}\right)$ определяется леммой 1 для компакта $G_{\gamma}$ и дифференциального включения $\dot{\gamma} \in G_{\gamma}$. По этой же лемме существует число $c_{0}, 0 \leqslant c_{0} \leqslant t_{\max }$, и решение $\beta_{0}=\beta_{0}(t)$ дифференциального включения $\dot{\gamma} \in G_{\gamma}$, определенное на отрезке $\left[t_{0}, t_{0}+c_{0}\right]$, такие, что

$$
\beta_{0}\left(t_{0}\right)=\gamma_{0}, \quad \beta_{0}\left(c_{0}\right)=\gamma_{0,0}+T_{\varepsilon, 0}
$$

для некоторого $\varepsilon$-почти периода $T_{\varepsilon, 0}$ функции $f\left(\tau_{0}, \cdot\right)$.

Теперь можно определить решение $\gamma_{*}$ на отрезке $\left[t_{0}, t_{1}\right]: \gamma_{*}(t)=\beta_{0}(t)$ при $t_{0} \leqslant t \leqslant$ $t_{0}+c_{0}, \gamma_{*}(t)=\gamma_{0}\left(t-c_{0}\right)+T_{\varepsilon, 0}$ при $t_{0}+c_{0} \leqslant t \leqslant t_{1}$.

Если решение $\gamma_{*}$ уже построено в промежутке $t_{0} \leqslant t \leqslant t_{j}, j \geqslant 1$, то оно продолжается на отрезок $\left[t_{j}, t_{j+1}\right]$ по следующей схеме.

По лемме 1 существуют постоянная $c_{j}, 0 \leqslant c_{j} \leqslant t_{\max }$, и решение $\beta_{j}=\beta_{j}(t)$ дифоференциального включения $\dot{\gamma} \in G_{\gamma}$, определенное на отрезке $\left[t_{j}, t_{j}+c_{j}\right]$, такие, что $\beta_{j}\left(t_{j}\right)=\gamma_{*}\left(t_{j}\right), \beta_{j}\left(c_{j}\right)=\gamma_{j, 0}+T_{\varepsilon, j}$ для некоторого $\varepsilon$-почти периода $T_{\varepsilon, j}$ функции $f\left(\tau_{j}, \cdot\right)$. 
Решение $\gamma_{*}$ на отрезке $\left[t_{j}, t_{j+1}\right]$ определяется следующим образом: $\gamma_{*}(t)=\beta_{j}(t)$ при $t_{j} \leqslant t \leqslant t_{j}+c_{j}, \gamma_{*}(t)=\gamma_{j}\left(t-c_{j}\right)+T_{\varepsilon, j}$ при $t_{j}+c_{j} \leqslant t \leqslant t_{j+1}$. Таким образом, решение $\gamma_{*} \in \Gamma_{\gamma}\left(\gamma_{0}\right)$ определено на всем промежутке $t \geqslant 0$.

Нетрудно видеть, что для построенного решения и решения $\gamma_{j}$ дифференциального включения $\dot{\gamma} \in G_{\gamma}, \gamma\left(t_{j}\right)=\gamma_{j, 0}$, имеет место оценка средних

$$
\left|\frac{1}{\Delta_{0}} \int_{t_{j}}^{t_{j+1}} f\left(\tau_{j}, \gamma_{j}(t)\right) d t-\frac{1}{\Delta_{0}} \int_{t_{j}}^{t_{j+1}} f\left(\tau_{j}, \gamma_{*}(t)\right) d t\right| \leqslant \frac{2 f_{0} t_{\max }}{\Delta_{0}}+\varepsilon=2 f_{0} t_{\max } \sqrt{\mu}+\varepsilon .
$$

На основании тождества

$$
\begin{aligned}
S_{n}\left(\tau_{*}\right)= & \frac{1}{n} \sum_{j=0}^{n-1} \frac{1}{\Delta_{0}} \int_{t_{j}}^{t_{j+1}} f\left(\tau_{j}, \gamma_{*}(t)\right) d t \\
= & \frac{1}{n} \sum_{j=0}^{n-1} \Phi\left(\tau_{j}\right)+\frac{1}{n} \sum_{j=0}^{n-1}\left(\frac{1}{\Delta_{0}} \int_{t_{j}}^{t_{j+1}} f\left(\tau_{j}, \gamma_{*}(t)\right) d t-\frac{1}{\Delta_{0}} \int_{t_{j}}^{t_{j+1}} f\left(\tau_{j}, \gamma_{j}(t)\right) d t\right) \\
& +\frac{1}{n} \sum_{j=0}^{n-1}\left(\frac{1}{\Delta_{0}} \int_{t_{j}}^{t_{j+1}} f\left(\tau_{j}, \gamma_{j}(t)\right) d t-\Phi\left(\tau_{j}\right)\right)
\end{aligned}
$$

и оценок $(13),(14)$ получим соотношение

$$
S_{n}\left(\tau_{*}\right) \geqslant \frac{1}{n \Delta_{0}} \sum_{j=0}^{n-1} \Phi\left(\tau_{j}\right) \Delta_{0}-2 f_{0} t_{\max } \sqrt{\mu}-\varphi\left(\frac{1}{\sqrt{\mu}}\right)-2 \varepsilon .
$$

Далее, согласно (11) при $\tau=\tau_{*}$ имеем

$$
S_{n}\left(\tau_{*}\right) \geqslant \frac{1}{n \Delta_{0}} \int_{0}^{n \Delta_{0}} \Phi\left(\tau_{*}(\mu t)\right) d t-2 f_{0} t_{\max } \sqrt{\mu}-\omega_{\tau}\left(\delta_{\mu}, f\right)-\varphi\left(\frac{1}{\sqrt{\mu}}\right)-2 \varepsilon .
$$

При достаточно большом $n \geqslant n_{1}(\varepsilon, \mu)$ и любых $\tau_{0} \in \mathbb{R}^{m_{\tau}}, \gamma_{0} \in \mathbb{R}^{m_{\gamma}}$ получим

$$
\left|\frac{1}{n \Delta_{0}} \int_{0}^{n \Delta_{0}} \Phi\left(\tau_{*}(\mu t)\right) d t-\Psi_{f}\right| \leqslant \varepsilon .
$$

Отсюда и из (15) следует требуемая оценка (9). Лемма 4 доказана.

Теперь переходим к непосредственному доказательству теоремы. По лемме 3 существует оптимальное решение $\tau_{\mathrm{op}}, \gamma_{\mathrm{op}}$ системы $(2),(3)$, для которого

$$
M_{f}(\mu)=\lim _{n \rightarrow \infty} M_{f}^{n}(\mu)
$$

где

$$
M_{f}^{n}(\mu)=\frac{1}{n \Delta_{0}} \int_{0}^{n \Delta_{0}} f\left(\tau_{\mathrm{op}}(\mu t), \gamma_{\mathrm{op}}(t)\right) d t
$$

Далее, поскольку

$$
M_{f}^{n}(\mu) \leqslant S_{n}\left(\tau_{\mathrm{op}}\right)+\omega_{\tau}\left(\delta_{\mu}, f\right),
$$


по лемме 4 из (8) при $\tau=\tau_{\text {ор }}$ следует

$$
M_{f}^{n}(\mu) \leqslant \Psi_{f}+2 \omega_{\tau}\left(\delta_{\mu}, f\right)+\varphi(1 / \sqrt{\mu})+\varepsilon
$$

С другой стороны, для любого фиксированного решения задачи (2), в частности для $\tau_{*} \in \Gamma_{\tau}\left(\tau_{0}\right)$, вьполняется неравенство

$$
M_{f}^{n}(\mu) \geqslant \sup _{\gamma} \frac{1}{n \Delta_{0}} \int_{0}^{n \Delta_{0}} f\left(\tau_{*}(\mu t), \gamma(t)\right) d t \geqslant S_{n}\left(\tau_{*}\right)-\omega_{\tau}\left(\delta_{\mu}, f\right) .
$$

В силу (9) имеем

$$
M_{f}^{n}(\mu) \geqslant \Psi_{f}-2 f_{0} t_{\max } \sqrt{\mu}-2 \omega_{\tau}\left(\delta_{\mu}, f\right)-\varphi\left(\frac{1}{\sqrt{\mu}}\right)-3 \varepsilon .
$$

После перехода к пределу в (16), (17) сначала при $n \rightarrow \infty$, а затем при $\mu \rightarrow+0$, получим соотношение $(5)$, так как функция $\varphi(1 / \sqrt{\mu})$ при $\mu \rightarrow+0$ является бесконечно малой по лемме 2 . Независимость $\Psi_{f}$ от начальных данных следует из невырожденности компактов $G_{\tau}, G_{\gamma}$ на основании леммы 2 . Теорема доказана.

5. Замечания. 1. В условиях теоремы множество $G_{\tau} \times G_{\gamma}$ может оказаться и вырожденным. Тогда предел максимального среднего $M_{f}$ в общем случае зависит от начальньх данных.

2. На примерах нетрудно показать, что вырожденность одного из компактов $G_{\tau}, G_{\gamma}$ может привести к зависимости предельного значения $\Psi_{f}$ от начальных данных. В то же время, предельное соотношение (5) будет иметь место и в этом более общем случае.

3. Для периодической функции $f: \mathbb{R}^{m_{\tau}} \times \mathbb{R}^{m_{\gamma}} \rightarrow \mathbb{R}$ при $m_{\gamma}=1$ из [1] следует, что $\varphi(1 / \sqrt{\mu})=O(\sqrt{\mu})$ при $\mu \rightarrow 0$, поэтому, если по переменной $\gamma \in \mathbb{R}$ функция $f$ будет липшищевой, то скорость сходимости к предельному значению в (5) будет не хуже, чем $\sqrt{\mu}$.

\section{СПИСОК ЦИТИРОВАННОЙ ЛИТЕРАТУРЫ}

[1] Филатов О.П. Вычисление пределов максимальных средних // Матем. заметки. 1996. T. 59. № 5. C. 759-767.

[2] Благодатских В.И., Филиппов А.Ф. Дифференциальные включения и оптимальное упрвление // Тр. МИАН СССР. 1985. Т. 169. С. 194-252.

[3] Васильев Ф. П. Численные методы решения экстремальных задач. М.: Наука, 1980. 